\title{
Torpedoschussmeldungen aus dem Zweiten Weltkrieg
}

Massenentsäuerung zur Bestandserhaltung historischer Quellen des Marinearchivs

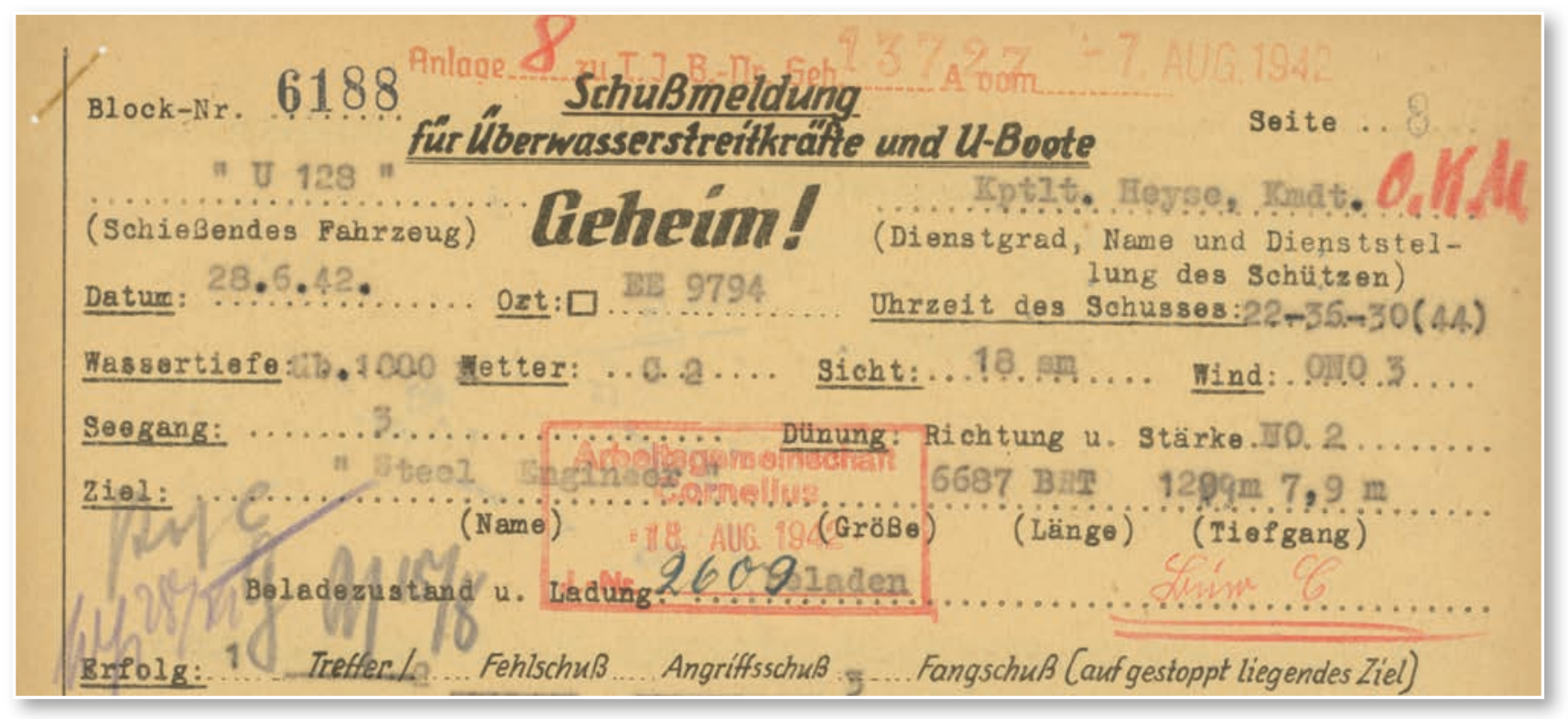

Abb. 1: Ausschnitt einer Torpedoschussmeldung von U 128

Aufgrund der Materialien, die bei der industriellen Papierherstellung zwischen 1850 und 1960 zum Einsatz kamen, tritt bei einem Großteil von Papier ein erhöhter Säuregehalt auf. Es verbräunt stark, verliert an Festigkeit und wird brüchig. Um diesen Zerfall zu stoppen, kann das Material entsäuert werden. Hierzu wird häufig das Verfahren der sogenannten Massenentsäuerung angewandt, bei dem der pH-Wert des Papiers möglichst neutralisiert und eine alkalische Reserve eingebracht wird. Bei dieser Reserve handelt es sich um eine Schutzfunktion, durch die eine erneute Säurebildung im Papier verhindert oder zumindest verzögert werden soll. Bereits entstandene Schäden können durch diese Technik jedoch nicht rückgängig gemacht werden. Um diesen irreversiblen Schäden vorzubeugen und Archivmaterialien oder Buchbestände des 19. und 20. Jahrhunderts zu erhalten, ist eine Entsäuerung daher oft unumgänglich. Da die Durchführung von Massenentsäuerungsprojekten in vielen Einrichtungen ansteht, haben die Konferenz der Leiterinnen und Leiter der Archivverwaltungen des Bundes und der Länder, die Bundes- konferenz der Kommunalarchive beim Deutschen Städtetag und der Deutsche Bibliotheksverband hierzu im Mai 2019 ein Grundlagenpapier veröffentlicht. ${ }^{1}$

Für die Bücher und die archivalischen Sammlungen der Bibliothek für Zeitgeschichte $(\mathrm{BfZ})$ in der Württembergischen Landesbibliothek besteht ein großer Handlungsbedarf, da ein erheblicher Teil der Bestände aus dem Zeitraum vom Ersten Weltkrieg bis in die 1960er-Jahre stammt. Zudem sind in dieser Abteilung auch zahlreiche Unikate und seltene Dokumente vorhanden, bei denen der Papierzerfall möglichst rasch gestoppt werden muss.

Aus dem Marinearchiv der BfZ wurden daher im Jahr 2019 für entsprechende Maßnahmen die Torpedoschussmeldungen deutscher U-Boote und Schiffe aus dem Zweiten Weltkrieg ausgewählt. Es handelt sich um einen abgeschlossenen Bestand, der mit einem Umfang von ungefähr 6.300 Einzelblättern (rund $31 \mathrm{~kg}$ ) in einer Tranche vollständig entsäuert werden konnte. Bei einer Vielzahl der Seiten sind bereits die typischen Schadensbilder von saurem Papier deutlich erkennbar. Neben der 
starken Verbräunung ist vor allem die Brüchigkeit des Materials besorgniserregend. Die Papiere kamen in den 1960er-Jahren über den langjährigen Direktor der BfZ, Jürgen Rohwer, in die Bibliothek. Dort dienten sie als wichtige Quelle für die Forschung zum Seekrieg 1939-1945. Mit Unterstützung des Landesrestaurierungsprogramms und des Instituts für Erhaltung von Archiv- und Bibliotheksgut wurde die Entsäuerung der Schussmeldungen bei der Firma Nitrochemie Aschau GmbH durchgeführt. Damit besteht jetzt die Möglichkeit, diese Dokumente für die Zukunft zu erhalten.
1946 lernte er ehemalig hohe deutsche Offiziere kennen, die im Naval Historical Team für die amerikanische Navy anhand des beschlagnahmten deutschen Aktenmaterials Marineoperationen analysierten. Über diese Kontakte arbeitete er als Student ab 1948 mit an einem offiziellen Werk der US Navy über den Seekrieg im Atlantik. Sein Interesse an der Wehrforschung und im Speziellen an der Geschichte des Seekrieges brachte Rohwer mit in die baden-württembergische Landeshauptstadt, bei der man aufgrund ihrer geographischen Lage nicht von vorneherein an einen marinehistorischen Forschungsstandort denkt.

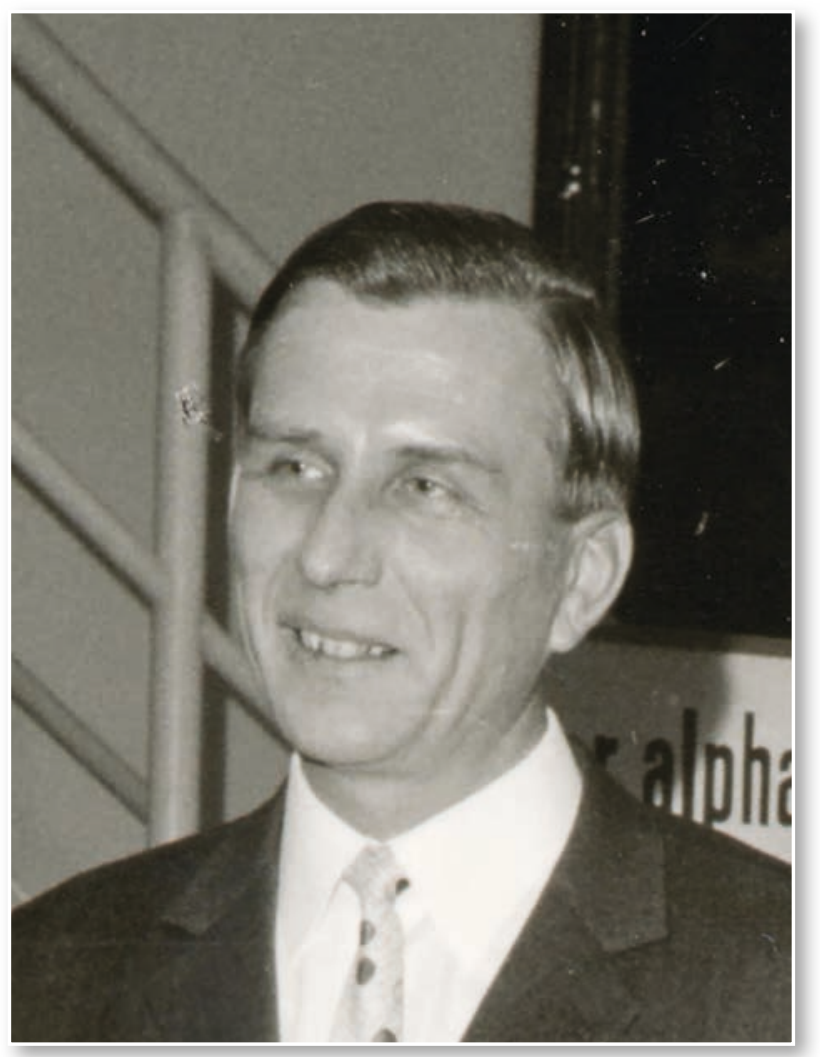

Abb. 3: Prof. Dr. Jürgen Rohwer 1965

Unter Rohwers Leitung entwickelte sich die marinegeschichtliche Sammlung der BfZ zu einer weltweit bekannten Anlaufstelle für Forscher und interessierte Laien. Dies lag neben dem fundierten Wissen des Personals sicherlich auch an der Vielzahl persönlicher Kontakte des Direktors zu Fachleuten in zum Teil höchsten Stellen im In- und Ausland. Durch diese Beziehungen konnte der Archivbestand der Bibliothek immer wieder um interessante Originaldokumente oder Kopien von sonst schwer zugänglichen Materialien ergänzt werden. Außerdem wurde damit begonnen, Fotos von Kriegsschiffen aus aller Welt zu sammeln, 
später erweitert um Fotografien von Handelsschiffen. Den Grundstock für das Marine-Fotoarchiv mit seinen inzwischen rund 500.000 Bildern legte Rohwer selbst mit seiner privaten Kollektion. Die vielschichtige Sammlung zur Marinegeschichte des 20. Jahrhunderts ist auch heute noch eine häufig frequentierte Informationsquelle der Bibliothek für Zeitgeschichte.

\section{Torpedoschussmeldungen}

Über welchen Weg die Torpedoschussmeldungen in die Marine-Sammlung der BfZ gelangten, kann heute nicht mehr mit letzter Sicherheit gesagt werden. Die Akten der Kriegsmarine waren bei Kriegsende erhalten geblieben und wurden durch die amerikanischen Streitkräfte beschlagnahmt, danach an die britische Admiralität überstellt. Durch Rohwers Arbeit im Naval Historical Team und später im Arbeitskreis für Wehrforschung ergaben sich weitere wertvolle Kontakte: so unter anderem auch zu Commander M.G. Saunders, dem Verwalter der deutschen Marineakten bei der britischen Admiralität. ${ }^{2}$ Im Vorwort zur 1999 erschienenen Neuauflage von Rohwers "Axis Submarine Successes of World War Two" findet sich ein Hinweis, dass die Schussmeldungen durch den zur British Admirality abgeordneten Amtsrat Pfeiffer bereitgestellt wurden. ${ }^{3}$

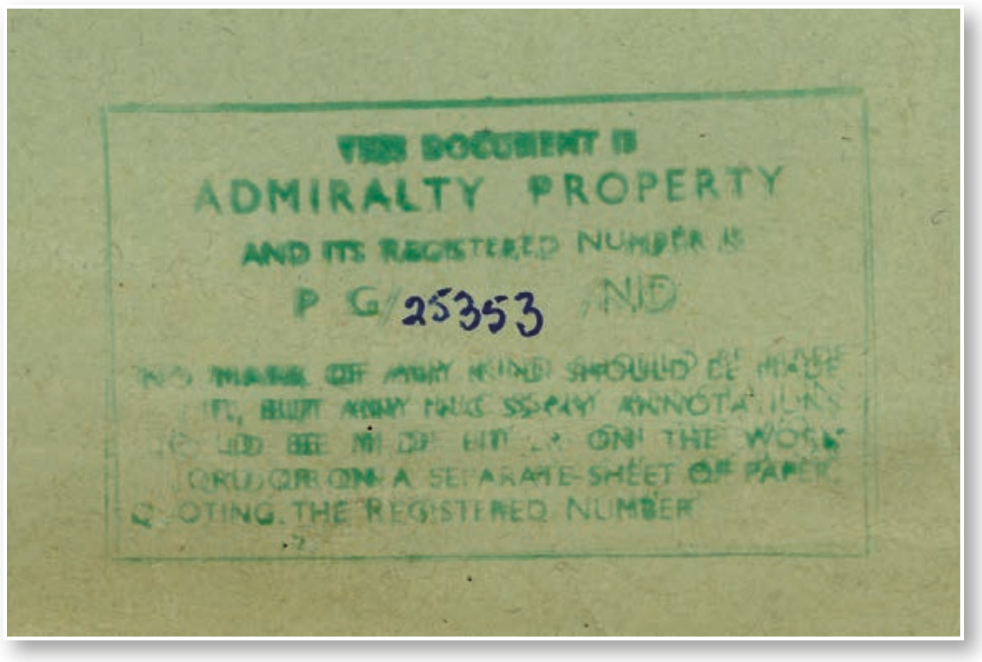

Abb. 4: Stempel der British Admirality mit PG-Nummer ${ }^{4}$

2) Vgl. Rohwer, Jürgen: "Vom Naval Historical Team zum Arbeitskreis für Wehrforschung", in: Hartmut Klüver, Thomas Weis (Hg.): Marinegeschichte, Seekrieg, Funkaufklärung, Düsseldorf 2004, S. 79-88, hier S. 84f.

3) Vgl. Rohwer, Jürgen: Axis submarine successes of World War Two, London 1999, vii.

4) Die PG-Nummern wurden bei der Erschließung des deutschen Aktenmaterials durch die British Admirality vergeben, um den Bestand zu ordnen. Die Buchstaben "PG" stehen angeblich für "pinched from the Germans", also für "den Deutschen abgenommen". Vgl. Burdick, Charles (1972): The Tambach Archive, in: Military Affairs 36 (4), S. 124-126, hier S. 125.

5) Vgl. hierzu Abb. 1.

6) Vgl. Bundesarchiv Militärarchiv Freiburg (BArch), TS 498/PG 41957: Schussauswertung der U-Boote, TVA/Gruppe Nowotny, 14.11.1945.
Eine Torpedoschussmeldung ist ein Formular mit vier Durchschlägen, welches nach jedem Torpedoangriff eines U-Bootes oder eines Schiffes ausgefüllt werden musste. Eine Ausfertigung wurde in das Kriegstagebuch des U-Bootes eingefügt, eine weitere erhielt der Befehlshaber der U-Boote (B.d.U.), Großadmiral Karl Dönitz. Die drei verbliebenen Exemplare gingen an die Torpedoversuchsanstalt Eckernförde, an das Amt Torpedowaffe beim Oberkommando der Kriegsmarine (OKM) und an die Torpedoinspektion. Die Schussmeldungen in der BfZ sind handschriftlich mit dem Vermerk OKM, also Oberkommando der Kriegsmarine, versehen. Somit handelt es sich um die Durchschläge, die an das Amt Torpedowaffe gingen. Dort wurden sie von der "Arbeitsgemeinschaft Cornelius" ausgewertet, auf die im Folgenden näher eingegangen wird. Dies ist anhand von Stempeln, handschriftlichen Anmerkungen und Kommentaren auf den einzelnen Formularen ersichtlich. ${ }^{5}$

In der BfZ befindet sich somit ein Satz aller erhaltenen Schussmeldungen der deutschen U-Boote und Überwassereinheiten. Wo die Sätze der Torpedoversuchsanstalt und der Torpedoinspektion geblieben sind und inwieweit sie den Krieg überhaupt überstanden haben, ist nicht bekannt. Die Kriegstagebücher der U-Boote und des B.d.U. sind zum größten Teil erhalten und die Originale befinden sich inzwischen im Bundesarchiv; Mikrofilm-Kopien dieser und weiterer wichtiger Quellen sind auch im Bestand der BfZ. Hier ist es möglich, die Schussmeldungen aufgrund ihrer chronologischen und nach U-Booten sortierten Ordnung unkompliziert zu nutzen.

\section{Die Torpedokrise 1940}

Die Kriegsmarine verwendete zu Beginn des Zweiten Weltkrieges den Torpedo G7 mit Elektro- oder Gasturbinenantrieb, der bereits im Ersten Weltkrieg eingesetzt und ab den 1920er-Jahren in der Torpedoversuchsanstalt Eckernförde weiterentwickelt worden war. Schon früh wurde deutlich, dass der G7 nur unzuverlässig funktionierte. Bei den ersten Feindfahrten der deutschen U-Boote im Jahr 1939 wurden zwar etliche Torpedos abgeschossen, aber erfolgreich waren die Angriffe selten. Eine Trefferquote von 33 Prozent $^{6}$ konnte das Oberkommando der Kriegsmarine nicht zufriedenstellen. Um die Gründe für die Unzuverlässigkeit der Torpedos aus- 
zumachen, wurde 1940 die Torpedoschussmeldung eingeführt. In dieser sollten die Zieleinstellungen am Torpedo, eine Beschreibung des Angriffsziels und der äußeren Bedingungen sowie die Beobachtung der Angriffs-Auswirkungen vermerkt werden. Die verschiedenen Forschungsanstalten hatten die Aufgabe, herauszufinden, ob ein Fehlschuss durch Fehleinschätzungen der Besatzung, aufgrund der Witterungsverhältnisse oder durch technisches Versagen begründet war. So sollte festgestellt werden, welche Verbesserungen am Torpedo nötig wären oder welche Zieleinstellungen z.B. bei der Tiefe zu vermeiden waren. im Anschluss an die "Weserübung" die gesamte Kriegsmarine bis hin zu ihrem Oberbefehlshaber.

Gegen die Mitarbeiter der Torpedoversuchsanstalt, die für den G7 verantwortlich waren, wurde ein Kriegsgerichtsverfahren eröffnet. Die Anklage bereitete sich sehr ausführlich auf das Verfahren vor, so dass die Verhandlung erst im Mai 1941 begann. Das Reichskriegsgericht stellte mehrere schwere Mängel bei der Entwicklung und Erprobung fest. So hatte man vor Kriegsbeginn nur zwei scharfe Testschüsse durchgeführt - 1933 und 1935. Diese Versäumnisse hätten „vorübergehend das Vertrau-

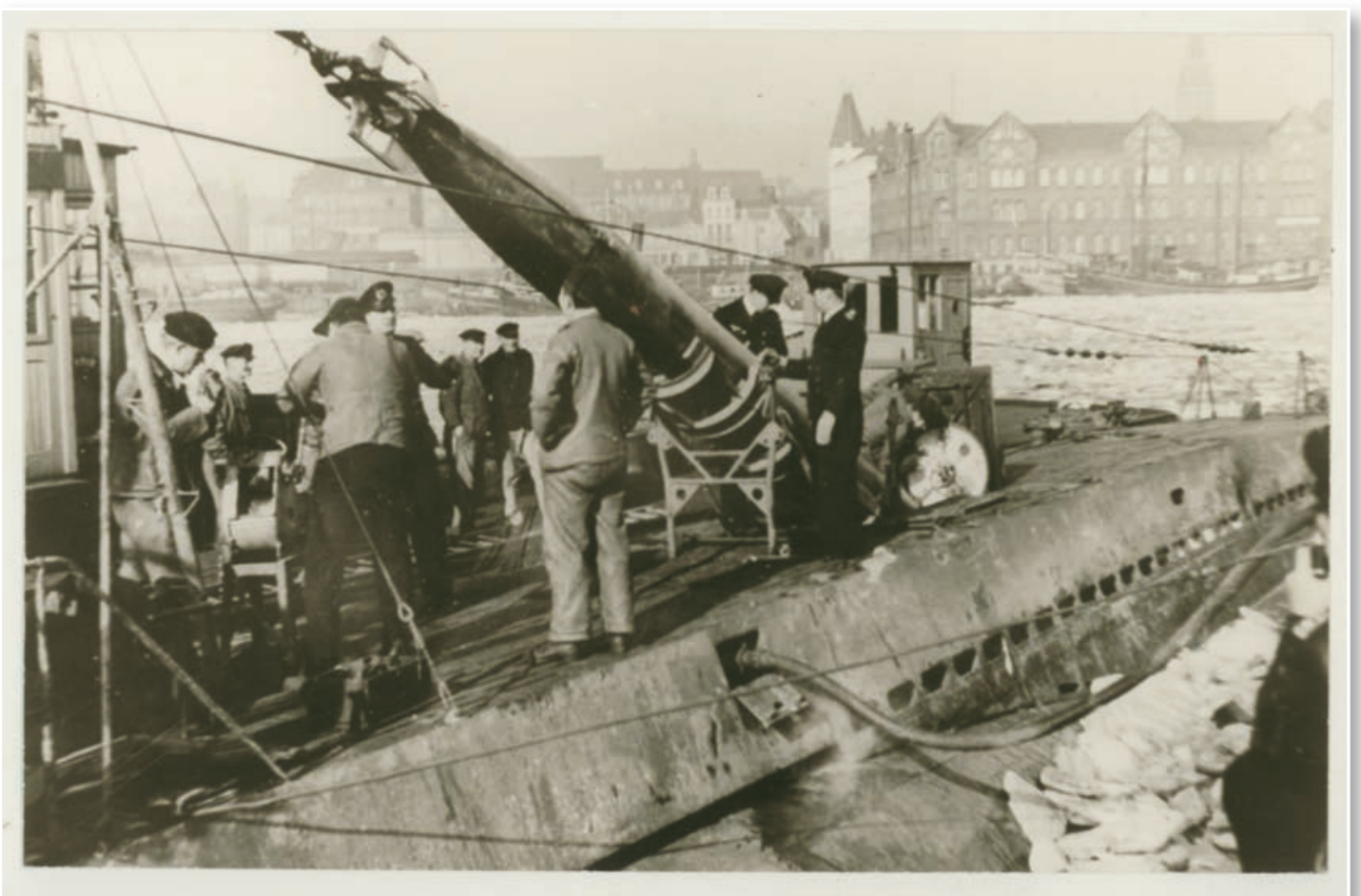

en der Front zur Torpedowaffe erschüttert, die Kriegsmarine um bedeutende Erfolge gebracht und den Gang der Kriegsereignisse nachteilig beeinflusst" . Der Prozess endete im Dezember mit Gefängnisstrafen für zwei Offiziere im Admirals-

Abb. 5: Torpedoübernahme durch ein U-Boot

Obwohl der Seekriegsleitung bekannt war, dass die Bewaffnung der Boote ein Problem darstellte, wurden U-Boote bei der ersten Großaktion der Kriegsmarine eingesetzt. Der Überfall auf Norwegen und Dänemark fand im April 1940 unter dem Decknamen „Weserübung" statt. Durch die Besetzung der beiden neutralen Länder wollte das Deutsche Reich den Seeweg über die Ostsee für die kriegswichtigen Erztransporte aus Nordnorwegen freihalten und eine Seeblockade durch Großbritannien verhindern. Es waren mehrere deutsche U-Boote beteiligt, die gegen die vor Norwegen operierenden britischen Einheiten eingesetzt werden sollten. Von allen abgefeuerten Torpedos versagten $66 \%$. Die sogenannte Torpedokrise beschäftigte rang sowie für zwei technische Beamte der TVA. Zwar kamen alle Angeklagten bereits nach sechs Monaten wieder frei, jedoch löste der Prozess bei der staatlichen Rüstungsindustrie große Aufregung aus.

Eine Arbeitsgemeinschaft zur Untersuchung der technischen Probleme war bereits zu Kriegsbeginn gegründet worden, da sich die Mängel durchaus schon 1939 abzeichneten. Kurioserweise wurde diese AG durch Ernst-August Cornelius geleitet, der mehrere Jahre maßgeblich die Verantwortung für die Entwicklung des G7 getragen hatte. Durch die systematische Auswertung der Schussmeldungen konnten schließlich einige technische Verbesserungen umgesetzt und verschiedene Richtlinien für die Zieleinstellung erstellt werden, wodurch 
die Anzahl der Fehlschüsse zurückging. Zeitgleich wurde auch die Entwicklung eines neuen Torpedomodells vorangetrieben, das zuverlässiger und mit einem neuen Zielfindungsmechanismus ausgestattet sein sollte. Beim sogenannten „ZaunkönigTorpedo" setzten die Entwickler anstelle von Aufschlags- oder Magnetzündern eine akustisch gesteuerte Zielfindung ein. Mit der Umstellung der Bewaffnung der U-Boote im März 1943 änderte sich auch das Verfahren für die Schussmeldungen. Statt Formulare mit mehreren Durchschlägen wurden nun maschinenlesbare Lochkarten-Schussmeldungen genutzt, um die Auswertung zu erleichtern.

\section{Klärung von Schiffsschicksalen in der Bibliothek für Zeitgeschichte}

Nach Ende des Zweiten Weltkriegs machten sich zahlreiche Historiker die akribisch geführten Unterlagen der Kriegsmarine zunutze. Jürgen Rohwer begann laut eigener Aussage bereits Ende der 1940er-Jahre mit der Arbeit zur Klärung von Schiffsschicksalen während des Krieges. Seine Hilfsmittel für die deutsche Seite waren anfangs die kurz nach dem Krieg hastig abgeschriebenen Unterlagen aus den damaligen deutschen Werften, die Kriegstagebücher des B.d.U und der Seekriegsleitung sowie die Torpedoschussmeldungen, die durch die British Admirality verfügbar wurden. Aber auch mit verschiedenen Stellen bei den anderen ehemaligen Achsenmächten entstanden im Laufe der Jahre eine gute Zusammenarbeit und ein reger Austausch von Dokumenten. Die alliierten Handelsschiffsverluste waren 1945 nach dem damaligen Stand der Kenntnisse in einer für den Dienstgebrauch vorgesehenen Liste „B.R. 1337: British and Foreign Merchant Vessel Lost or Damaged by Enemy Action During Second World War" verzeichnet, auf die zugegriffen werden konnte. Die Verluste der alliierten Kriegsschiffe wurden ebenfalls von der britischen Admiralität erfasst. Zu diesen Angaben führt die Bibliothek für Zeitgeschichte eine sogenannte Schiffsverlustkartei.

Über viele Jahre konnte so ein großer Teil von bis dahin ungeklärten Schiffsschicksalen ermittelt werden. Hierzu bedurfte es einer stetigen Korrektur und Aktualisierung der Daten. Damit auch andere

8) Rohwer, Jürgen: Die U-Boot-Erfolge der Achsenmächte 1939-1945,

9) Rohwer, Jürgen: Axis submarine successes of World War Two, London 1999.

10) kurz für: Axis Submarine Successes

11) Webseite unter: www.historisches-marinearchiv.de

12) Webseite unter: www.forum-marinearchiv.de

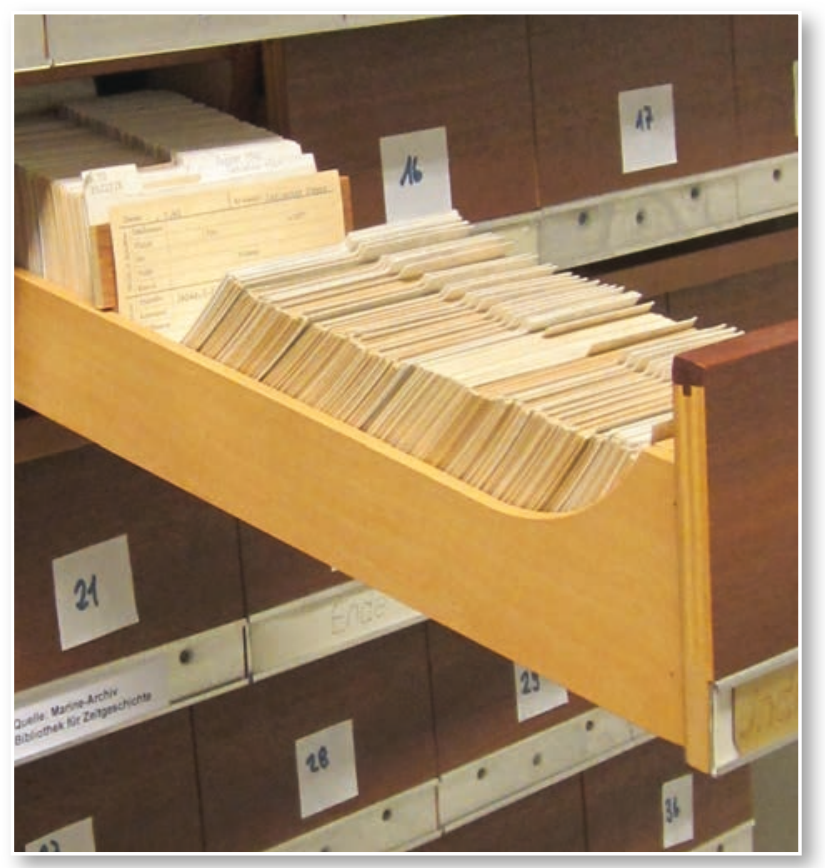

Abb. 6: Die Schiffsverlustkartei im Marinearchiv

Institutionen weltweit diese Forschungsergebnisse nutzen konnten, erschien 1968 die erste Auflage von "Die U-Booterfolge der Achsenmächte" ${ }^{\text {. }}$. Darin findet sich eine nach Kriegsschauplätzen getrennte chronologische Auflistung aller U-Boot-Angriffe auf alliierte Schiffe. Aufgrund des internationalen Interesses folgten zwei überarbeitete und korrigierte Neuauflagen in englischer Sprache, die letzte $1999^{9}$.

Mit der Verbreitung des Internets bot es sich an, die Schiffsverlustkartei online zu stellen, da Korrekturen und Ergänzungen noch immer nötig sind. Die ASS ${ }^{10}$-Datenbank wird auf der Website des Historischen Marinearchivs (HMA) ${ }^{11}$ gehostet, wo auch weitere marinehistorische Datenbanken angeboten werden. Korrekturen und Datenpflege werden hier durch Thomas Weis, der von 1992-2018 das Marinearchiv der BfZ betreute, und den Mitgliedern des "Forum Marinearchiv" (FMA)12 durchgeführt.

Der Umfang der verschiedenen Quellen bedeutete schon zu Beginn der Schiffsverlustkartei eine große Herausforderung und auch heute werden die Originaldokumente nach wie vor im Zweifelsfall für Überprüfungen herangezogen. Deshalb ist es wichtig, Bestandserhaltungsmaßnahmen wie im Falle der Torpedoschussmeldungen durchzuführen, solange das Material noch nicht zu stark in Mitleidenschaft gezogen ist.

Tobias Thelen 